\title{
Workshop: consumption and consumer perceptions
}

\author{
Don Stewart* \\ 13 Venner Road, London SE26 5EQ, UK
}

Consumer: Polyols: Symptoms: Sugar-free: Labelling

There is relatively little published information regarding consumer perceptions of the possible health benefits or occurrence of gastrointestinal symptoms following consumption of low-digestible carbohydrates (LDCs), in particular polyol sugars. This workshop focused on how consumers perceive the possible health benefits of LDCs, their reactions to any 'adverse' symptoms following LDC consumption and how the medical profession, regulatory bodies, health educators and manufacturers might respond. It was also discussed if observations made in the laboratory were indeed relevant to the wider community. Although the workshop mainly discussed polyol sugars compared to other LDCs it was concluded that the functional benefits of LDC consumption far outweighed any intolerance effects and that the majority of consumer reactions and perceptions support this view.

\section{Consumer studies}

Specialist marketing companies have conducted studies over a number of years to investigate consumer reactions to polyol-containing products. These products have been representative of currently available polyol products, e.g. sugar-free/sugar reduced chocolate, sweets and candies. The market research tolerance studies performed have often been similar in design to the double-blind, cross-over protocols now recommended for laboratory-based investigations. However, these studies have comprised much larger samples (e.g. 1000 individuals) and have attempted to create a consumer friendly environment by allowing respondents to consume products in their own homes as part of their general diet and as far as possible, describe any intolerance effects in their own terms.

These studies have consistently indicated that consumption of polyol-containing products is associated with a greater reporting of gastrointestinal symptoms (e.g. colic, bloating, flatus, diarrhoea) compared to control products containing sucrose. These reactions have rarely been described as serious or as lasting more than an hour or so. No survey has ever indicated that any adverse reaction or gastrointestinal effect was serious enough to require medical attention.

One marketing survey was designed to establish the proportion of the population claiming to be allergic to certain foods or food ingredients or experiencing any adverse reactions following consumption of sugar-free confectionery in normal consuming circumstances (SRU, 1992). Some major findings of this study were as follows.

(1) Five $(0.5 \%)$ respondents claimed to be sensitive or allergic to sugar-free products.

(2) When prompted, a further 25 respondents $(2.5 \%)$ reported having experienced mild adverse reactions at some time, although all still consumed sugar-free products.

(3) Two respondents $(0.2 \%)$ no longer consumed sugarfree products because of previous 'experience' of adverse reactions following their consumption.

\section{Perceived benefits of sugar-free consumption}

Consumer awareness of the benefits of sugar-free and LDC consumption (especially in more health-conscious individuals) is increasing. However, consumer perceptions of the benefits of LDC consumption can be vague and often confused with other products and food claims, e.g. 'light' etc. This demonstrates the need to educate the consumer further as to the real benefits of LDC consumption. One study has shown that consumers tend to perceive the benefit of sugar-free products as toothfriendly, i.e. non-cariogenic, not having the negative effects of sugar, and in some cases as being a dietary aid (SRU, 1992).

\section{The case against sugar-free}

The case against sugar-free at the consumer level tends to be seen in terms of taste and a 'pro-sugar sentiment' with little evidence of consumer awareness of the potential occurrence of gastrointestinal symptoms following sugarfree consumption. In some studies consumers prefer the taste of conventional sucrose-containing products compared to alternative polyl-based sugar-free products. In the US and the UK sugar producers have promoted the 'naturalness' of sugar and its energy-giving properties. Furthermore, market studies have found evidence of some consumers who care little whether or not sugar products are good or bad for them and continue to consume sugarcontaining products because they like them. Changing the

\footnotetext{
* Corresponding author: Don Stewart, fax +44 (0)20 8659 3144, email dmst@waitrose.com
} 
perception and/or habits of such consumers in favour of reduced sugar consumption has not been helped by a prevailing anti-science mood in the European Community. Many consumers see science as no longer having society's best interests at heart, examples being controversy over genetically modified (GM) foods and bovine spongiform encephalopathy (BSE). This has led to consumer distrust of what is perceived to be 'artificial', however broadly or narrowly defined.

\section{Labelling}

There was a strong view during the workshop that polyolcontaining products are discriminated against. Products containing relatively low levels of polyol are required to advertise the potential laxative effect whereas other products with similar potential are not. Furthermore, gastrointestinal symptoms following consumption of foods such as beans and artichokes are potentially just as uncomfortable and/or socially embarrassing. However, these gastrointestinal (GI) effects are usually taken for granted. The workshop considered that advertising a potential laxative effect may create an environment where GI symptoms more readily occur and deter some consumers from benefiting from the functional properties that polyol-containing products have to offer.

On the other hand, responsible manufacturers do not wish to conceal or be considered as concealing relevant information from consumers. In the rare cases that GI symptoms have prompted customer complaints (usually a mother after her young child has experienced GI symptoms), an explanation of how polyols function tends to assuage any anger. Often such consumers request that the potential to cause GI symptoms be made clearer or larger on product packaging. The workshop concluded that the conundrum should be resolved by educating the consumer rather than employing emotive warning labels.

\section{Consumer education}

The workshop suggested that consumer education should focus on the benefits of polyols and other LDCs with respect to their reduced cariogenicity, reduced glycaemia, reduced energy value, and other demonstrable beneficial effects to the gastrointestinal tract. Members of the medical profession and health educators should also be made more aware of these facts. Possible gastrointestinal symptoms should be placed in an appropriate context in that they should not be considered as a sign of illness or disease but rather as a transient GI response.

\section{Reference}

SRU (1992) Consumer Food Tolerance Survey, Switzerland. London: SRU Ltd. 